\title{
Fourth power mean of character sums
}

by

\section{Zhefeng Xu and Wenpeng Zhang (Xi'an)}

1. Introduction. Let $q \geq 3$ be an integer and $\chi$ be a Dirichlet character modulo $q$. The character sums

$$
\sum_{a=N+1}^{N+H} \chi(a)
$$

play an important role in number theory. Pólya [5] and Vinogradov [6] proved the inequality

$$
\left|\sum_{a=1}^{x} \chi(a)\right| \leq c \sqrt{p} \ln p
$$

when $q=p$ is a prime. Actually, the above inequality holds with the constant $c=1$. D. A. Burgess [1] obtained the mean value estimate

$$
\sum_{n=1}^{k}\left|\sum_{m=1}^{h} \chi(n+m)\right|^{2}<k h,
$$

where $h$ is any positive integer. For fourth power moments, he specified the problem to the case of $q=p$, and proved (see [2])

$$
\sum_{\chi \neq \chi_{0}} \sum_{n=1}^{p}\left|\sum_{m=1}^{h} \chi(n+m)\right|^{4} \leq 6 p^{2} h^{2} .
$$

For general modulus $q$, he summed the mean value over all primitive characters and obtained (see [3])

$$
\sum_{\chi \bmod }^{*} \sum_{q=1}^{p}\left|\sum_{m=1}^{h} \chi(n+m)\right|^{4} \leq 8 \tau^{7}(q) q^{2} h^{2},
$$

2000 Mathematics Subject Classification: Primary 11L40.

Key words and phrases: character sums, quarter intervals, fourth power mean, Dedekind sums, Cochrane sums.

This work is supported by NWU Doctorate Dissertation of Excellence Funds (07YYB03) and the N.S.F. (10671155) of P.R. China. 
where $\sum_{\chi \bmod q}^{*}$ denotes the summation over all primitive characters modulo $q$ and $\tau(n)$ is the Dirichlet divisor function.

In order to obtain an asymptotic formula for higher moments of character sums, the authors [7] studied the $2 k$ th power mean of the even primitive character sums over the quarter interval $[1, q / 4)$, and obtained the following sharper asymptotic formula:

$$
\begin{aligned}
\sum_{\chi(-1)=1}^{*} & \left|\sum_{a<q / 4} \chi(a)\right|^{2 k} \\
& =\frac{J(q) q^{k}}{16}\left(\frac{\pi}{8}\right)^{2 k-2} \prod_{p \mid q}\left(1-\frac{1}{p^{2}}\right)^{2 k-1} \prod_{p \nmid 2 q}\left(1-\frac{1-C_{2 k-2}^{k-1}}{p^{2}}\right)+O\left(q^{k+\varepsilon}\right),
\end{aligned}
$$

where $\sum_{\chi(-1)=1}^{*}$ denotes the summation over all primitive characters modulo $q$ such that $\chi(-1)=1, \varepsilon$ is any fixed positive number, $J(q)$ denotes the number of primitive characters modulo $q$, and $\prod_{p \mid q}$ denotes the product over all prime divisors $p$ of $q$; finally $C_{m}^{n}=m ! / n !(m-n) !$.

Unfortunately, the methods used in [7] only work for the case of primitive characters. For a general nonprincipal character modulo $q$, they are not efficient.

The present work deals mainly with the fourth power mean of the nonprincipal character sums over the interval $[1, q / 4)$ by using the properties of Dedekind sums, Cochrane sums and Dirichlet L-functions, and obtains a sharper asymptotic formula for it. We prove the following:

Theorem. Let $q \geq 5$ be an odd integer. Then we have the asymptotic formula

$$
\sum_{\chi \neq \chi_{0}}\left|\sum_{a<q / 4} \chi(a)\right|^{4}=\frac{21 \phi^{4}(q)}{256 q} \prod_{p^{\alpha} \| q} \frac{\frac{(p+1)^{3}}{p\left(p^{2}+1\right)}-\frac{1}{p^{3 \alpha-1}}}{1+\frac{1}{p}+\frac{1}{p^{2}}}+O\left(q^{2+\varepsilon}\right),
$$

where $\varepsilon$ is any fixed positive number and $\prod_{p^{\alpha} \| q}$ denotes the product over all prime divisors $p$ of $q$ with $p^{\alpha} \mid q$ and $p^{\alpha+1} \nmid q$.

For $k \geq 3$, how to get an asymptotic formula for $\sum_{\chi \neq \chi_{0}}\left|\sum_{a<q / 4} \chi(a)\right|^{2 k}$ is an open problem.

2. Some lemmas. To prove the theorem, we need the following lemmas.

Lemma 1 (see [4]). Let $q \geq 3$ be an odd number. For any nonprincipal character $\chi$ modulo $q$, we have

$$
\sum_{a=1}^{q} a \chi(a)=\frac{\chi(2) q}{1-2 \chi(2)} \sum_{a=1}^{(q-1) / 2} \chi(a) .
$$


Lemma 2 ([7, proof of Lemma 3]). Let $q \geq 5$ be an odd integer and $\chi$ be a Dirichlet character modulo $q$ such that $\chi(-1)=1$. Then

$$
\sum_{a=1}^{[q / 4]} \chi(a)=-\frac{\bar{\chi}(4)}{8 q} \sum_{a=1}^{4 q} a \chi \chi_{4}(a),
$$

where $\chi_{4}$ is the primitive Dirichlet character modulo 4.

Lemma 3. Let $q$ be an odd number and $\chi$ be a primitive Dirichlet character modulo $q$ such that $\chi(-1)=-1$. Then

$$
\sum_{a=1}^{[q / 4]} \chi(a)=\frac{\bar{\chi}(4)-\bar{\chi}(2)-2}{2 q} \sum_{a=1}^{q} a \chi(a) .
$$

Proof. We only prove the lemma in the case of $q \equiv 1(\bmod 4)$. A similar argument yields the same result for $q \equiv 3(\bmod 4)$. From the properties of the Dirichlet character modulo $q$, we can write

$$
\begin{aligned}
\text { (1) } & 4 \chi(4) \sum_{a=1}^{q-1} a \chi(a) \\
= & \sum_{a=1}^{(q-1) / 4} 4 a \chi(4 a)+\sum_{a=(q+3) / 4}^{(2 q-2) / 4} 4 a \chi(4 a)+\sum_{a=(2 q+2) / 4}^{(3 q-3) / 4} 4 a \chi(4 a)+\sum_{a=(3 q+1) / 4}^{q-1} 4 a \chi(4 a) \\
= & \sum_{a=1}^{(q-1) / 4} 4 a \chi(4 a)+\sum_{a=1}^{(q-1) / 4}(4 a+q-1) \chi(4 a-1) \\
& +\sum_{a=1}^{(q-1) / 4}(4 a+2 q-2) \chi(4 a-2)+\sum_{a=1}^{(q-1) / 4}(4 a+3 q-3) \chi(4 a-3) \\
= & \sum_{a=1}^{q-1} a \chi(a)+\chi(4) q \sum_{a=1}^{(q-1) / 4} \chi(a-\overline{4}) \\
& +2 \chi(4) q \sum_{a=1}^{(q-1) / 4} \chi(a-2 \cdot \overline{4})+3 \chi(4) q \sum_{a=1}^{(q-1) / 4} \chi(a-3 \cdot \overline{4}) .
\end{aligned}
$$

Note that $\overline{4} \equiv \frac{3 q+1}{4}(\bmod q)$ if $q \equiv 1(\bmod 4)$. So from $(1)$, we have

$$
\begin{aligned}
4 \chi(4) \sum_{a=1}^{q-1} a \chi(a)= & \sum_{a=1}^{q-1} a \chi(a)-\chi(4) q \sum_{a=(2 q+2) / 4}^{(3 q-3) / 4} \chi(a) \\
& -2 \chi(4) q \sum_{a=(q+3) / 4}^{(2 q-2) / 4} \chi(a)-3 \chi(4) q \sum_{a=1}^{(q-1) / 4} \chi(a)
\end{aligned}
$$




$$
\begin{aligned}
& =\sum_{a=1}^{q-1} a \chi(a)-\chi(4) q \sum_{a=(q+3) / 4}^{(2 q-2) / 4} \chi(a)-3 \chi(4) q \sum_{a=1}^{(q-1) / 4} \chi(a) \\
& =\sum_{a=1}^{q-1} a \chi(a)-\chi(4) q \sum_{a=1}^{(q-1) / 2} \chi(a)-2 \chi(4) q \sum_{a=1}^{(q-1) / 4} \chi(a),
\end{aligned}
$$

where we have used the equality $\chi(-1)=-1$ and

$$
\sum_{a=(q+3) / 4}^{(2 q-2) / 4} \chi(a)=-\sum_{a=(2 q+2) / 4}^{(3 q-3) / 4} \chi(a)
$$

Now, from (2) and Lemma 1, we get

$$
\begin{aligned}
& 4 \chi(4) \sum_{a=1}^{q-1} a \chi(a) \\
& \quad=\sum_{a=1}^{q-1} a \chi(a)-(\chi(2)-2 \chi(4)) \sum_{a=1}^{q-1} a \chi(a)-2 \chi(4) q \sum_{a=1}^{(q-1) / 4} \chi(a) .
\end{aligned}
$$

That is,

$$
\sum_{a=1}^{(q-1) / 4} \chi(a)=\frac{\bar{\chi}(4)-\bar{\chi}(2)-2}{2 q} \sum_{a=1}^{q-1} a \chi(a)=\frac{\bar{\chi}(4)-\bar{\chi}(2)-2}{2 q} \sum_{a=1}^{q} a \chi(a) .
$$

This completes the proof of Lemma 3 in the case of $q \equiv 1(\bmod 4)$.

Lemma 4 (see [8]). Let $q \geq 3$ be an integer with $(h, q)=1$. Denote by $S(h, q)$ the Dedekind sum

$$
S(h, q)=\sum_{a=1}^{q}\left(\left(\frac{a}{q}\right)\right)\left(\left(\frac{h a}{q}\right)\right),
$$

where

$$
((x))= \begin{cases}x-[x]-1 / 2 & \text { if } x \text { is not an integer } \\ 0 & \text { if } x \text { is an integer. }\end{cases}
$$

Then

$$
S(h, q)=\frac{1}{\pi^{2} q} \sum_{d \mid q} \frac{d^{2}}{\phi(d)} \sum_{\substack{\chi \bmod d \\ \chi(-1)=-1}} \chi(h)|L(1, \chi)|^{2},
$$

where the last sum is over all Dirichlet character modulo d with $\chi(-1)=-1$, and $L(s, \chi)$ is the Dirichlet L-function corresponding to $\chi$. 
Lemma 5. Let $q$ be any odd integer with $q \geq 3$ and $\chi$ be a Dirichlet character modulo $q$. Then for any integer $m \geq 0$, we have the identity

$$
\begin{aligned}
& \sum_{\substack{\chi \bmod q \\
\chi(-1)=-1}} \bar{\chi}\left(2^{m}\right)\left|\sum_{r=1}^{q} r \chi(r)\right|^{4} \\
& \quad=q^{4} \phi(q) \sum_{d \mid q} \sum_{l \mid q} \mu(d) \mu(l) \sum_{r=1}^{q} S\left(2^{m} r, q / d\right) S(r, q / l),
\end{aligned}
$$

where $\mu(n)$ is the Möbius function and the last sum is over all integers $r$ with $1 \leq r \leq q$ and $(r, q)=1$.

Proof. First we define the Cochrane sum $C(h, q)$, which was first introduced by Professor Todd Cochrane during his visit to Xi'an in October 2000, as follows:

$$
C(h, q)=\sum_{a=1}^{q}\left(\left(\frac{\bar{a}}{q}\right)\right)\left(\left(\frac{a h}{q}\right)\right) .
$$

Note that

$$
\sum_{c=1}^{q} \chi(c)\left(\left(\frac{c}{q}\right)\right)=\sum_{c=1}^{q} \chi(c)\left(\frac{c}{q}-\frac{1}{2}\right)=0 \quad \text { if } \chi(-1)=1 .
$$

Then from the orthogonality relation for Dirichlet characters modulo $q$, we can write

$$
\begin{aligned}
C(a, q) & =\sum_{r=1}^{q}\left(\left(\frac{\bar{r}}{q}\right)\right)\left(\left(\frac{a r}{q}\right)\right) \\
& =\frac{1}{\phi(q)} \sum_{\chi \bmod q}\left\{\sum_{r=1}^{q} \chi(r)\left(\left(\frac{r}{q}\right)\right)\right\} \times\left\{\sum_{s=1}^{q} \chi(s)\left(\left(\frac{a s}{q}\right)\right)\right\} \\
& =\frac{1}{\phi(q)} \sum_{\substack{\chi \bmod q \\
\chi(-1)=-1}}\left\{\sum_{r=1}^{q} \chi(r)\left(\left(\frac{r}{q}\right)\right)\right\} \times\left\{\sum_{s=1}^{q} \bar{\chi}(a) \chi(a s)\left(\left(\frac{a s}{q}\right)\right)\right\} \\
& =\frac{1}{\phi(q)} \sum_{\substack{\chi \bmod q \\
\chi(-1)=-1}} \bar{\chi}(a)\left(\sum_{r=1}^{q} \chi(r)\left(\left(\frac{r}{q}\right)\right)\right)^{2} \\
& =\frac{1}{q^{2} \phi(q)} \sum_{\substack{\chi \bmod q \\
\chi(-1)=-1}} \bar{\chi}(a)\left(\sum_{r=1}^{\prime} r \chi(r)\right)^{2},
\end{aligned}
$$

where we have used the fact that $\sum_{r=1}^{q} \chi(r)=0$ if $\chi$ is not the principal 
character modulo $q$. Now the identity

$$
\sum_{a=1}^{q} C\left(2^{m} a, q\right) C(a, q)=\frac{1}{q^{4} \phi(q)} \sum_{\substack{\chi \bmod q \\ \chi(-1)=-1}} \bar{\chi}\left(2^{m}\right)\left|\sum_{r=1}^{q} r \chi(r)\right|^{4}
$$

follows from (3) immediately.

On the other hand, from the definition of $C(a, q)$ we have

$$
\begin{aligned}
\sum_{a=1}^{q} C\left(2^{m} a, q\right) C(a, q) & =\sum_{a=1}^{q} \sum_{r=1}^{q}\left(\left(\frac{\bar{r}}{q}\right)\right)\left(\left(\frac{2^{m} a r}{q}\right)\right) \sum_{s=1}^{q}\left(\left(\frac{\bar{s}}{q}\right)\right)\left(\left(\frac{a s}{q}\right)\right) \\
& =\sum_{r=1}^{q} \sum_{s=1}^{\prime}\left(\left(\frac{\bar{r}}{q}\right)\right)\left(\left(\frac{\bar{s}}{q}\right)\right) \sum_{a=1}^{q}\left(\left(\frac{2^{m} a r}{q}\right)\right)\left(\left(\frac{a s}{q}\right)\right) \\
& =\sum_{r=1}^{q} \sum_{s=1}^{\prime}\left(\left(\frac{\bar{r}}{q}\right)\right)\left(\left(\frac{\bar{s}}{q}\right)\right) \sum_{a=1}^{q}\left(\left(\frac{2^{m} r \bar{s} a}{q}\right)\right)\left(\left(\frac{a}{q}\right)\right) .
\end{aligned}
$$

In the last step we have used the fact that if $(s, q)=1$ and $a$ runs through a reduced residue system modulo $q$, so does $\bar{s} a$. Therefore, from the definition of Dedekind sums $S(h, q)$ and the identities

$$
\sum_{s=1}^{q}=\sum_{d \mid q} \mu(d) \sum_{s=1}^{q / d} \text { and } S(r, q)=S(\bar{r}, q),
$$

we have

$$
\begin{aligned}
\sum_{a=1}^{q} C\left(2^{m}\right. & a, q) C(a, q) \\
& =\sum_{r=1}^{q} \sum_{s=1}^{\prime}\left(\left(\frac{\bar{r}}{q}\right)\right)\left(\left(\frac{\bar{s}}{q}\right)\right) \sum_{a=1}^{q}\left(\left(\frac{2^{m} r \bar{s} a}{q}\right)\right)\left(\left(\frac{a}{q}\right)\right) \\
& =\sum_{r=1}^{q} \sum_{s=1}^{\prime}\left(\left(\frac{\bar{r}}{q}\right)\right)\left(\left(\frac{\bar{s}}{q}\right)\right) \sum_{l \mid q} \mu(l) \sum_{a=1}^{q / l}\left(\left(\frac{2^{m} r \bar{s} a}{q / l}\right)\right)\left(\left(\frac{a}{q / l}\right)\right) \\
& =\sum_{l \mid q} \mu(l) \sum_{r=1}^{q} \sum_{s=1}^{\prime}\left(\left(\frac{\bar{r}}{q}\right)\right)\left(\left(\frac{\bar{s}}{q}\right)\right) S\left(2^{m} r \bar{s}, q / l\right) \\
& =\sum_{l \mid q} \mu(l) \sum_{r=1}^{q} \sum_{s=1}^{q}\left(\left(\frac{\bar{r}}{q}\right)\right)\left(\left(\frac{s}{q}\right)\right) S\left(2^{m} r s, q / l\right) \\
& =\sum_{l \mid q} \mu(l) \sum_{d \mid q} \mu(d) \sum_{r=1}^{\prime} \sum_{s=1}^{q / d}\left(\left(\frac{2^{m} r s}{q / d}\right)\right)\left(\left(\frac{s}{q / d}\right)\right) S(\bar{r}, q / l)
\end{aligned}
$$




$$
\begin{aligned}
& =\sum_{d \mid q} \sum_{l \mid q} \mu(d) \mu(l) \sum_{r=1}^{q} S\left(2^{m} r, q / d\right) S(\bar{r}, q / l) \\
& =\sum_{d \mid q} \sum_{l \mid q} \mu(d) \mu(l) \sum_{r=1}^{q} S\left(2^{m} r, q / d\right) S(r, q / l) .
\end{aligned}
$$

Now Lemma 5 follows from (4) and (5).

LEMMA 6. Let $u$ and $v$ be odd integers with $(u, v)=d \geq 2$, and $\chi_{u}^{0}$ and $\chi_{v}^{0}$ be the principal characters modulo $u$ and $v$, respectively. If $r(n)=$ $\sum_{d \mid n} \chi_{u}^{0}(d) \chi_{v}^{0}(n / d)$, then for any integer $m \geq 0$ we have the identity

$$
\sum_{\substack{n=1 \\(n, d)=1}}^{\infty} \frac{r\left(2^{m} n\right) r(n)}{n^{2}}=\frac{(3 m+5) \pi^{4}}{72} \frac{\prod_{p \mid u v} \frac{\left(p^{2}-1\right)^{2}}{p^{2}\left(p^{2}+1\right)}}{\prod_{p \mid d} \frac{p^{2}}{p^{2}-1}}
$$

Proof. Noting that $r(n)$ is a multiplicative function, we can write

$$
\begin{aligned}
& \sum_{\substack{n=1 \\
(n, d)=1}}^{\infty} \frac{r\left(2^{m} n\right) r(n)}{n^{2}} \\
& =\left(r\left(2^{m}\right)+\sum_{j=1}^{\infty} \frac{(m+j+1)(j+1)}{4^{j}}\right) \sum_{\substack{n=1 \\
(n, d)=1 \\
2 \nmid n}}^{\infty} \frac{r^{2}(n)}{n^{2}} .
\end{aligned}
$$

After some simple calculation, we get

$$
\sum_{j=1}^{\infty} \frac{(m+j+1)(j+1)}{4^{j}}=\frac{21 m+53}{27} .
$$

Moreover,

$$
\sum_{\substack{n=1 \\(n, d)=1 \\ 2 \nmid n}}^{\infty} \frac{r^{2}(n)}{n^{2}}=\prod_{\substack{p \nmid d \\ p \neq 2}}\left(1+\frac{r^{2}(p)}{p^{2}}+\frac{r^{2}\left(p^{2}\right)}{p^{4}}+\cdots\right)
$$

by using the Euler product formula. Note that

$$
r\left(p^{\alpha}\right)= \begin{cases}1 & \text { if } p \mid u, p \nmid v, \\ \alpha+1 & \text { if } p \nmid u, p \nmid v, \\ 1 & \text { if } p \nmid u, p \mid v, \\ 0 & \text { if } p|u, p| v,\end{cases}
$$


for any positive integer $\alpha$ and prime $p$. Hence,

$$
\begin{aligned}
\sum_{\substack{n=1 \\
(n, d)=1 \\
2 \nmid n}}^{\infty} \frac{r^{2}(n)}{n^{2}}= & \prod_{\substack{p \nmid u v \\
p \neq 2}}\left(1+\frac{2^{2}}{p^{2}}+\frac{3^{2}}{p^{4}}+\cdots\right) \\
& \times \prod_{\substack{p \mid u \\
p \nmid v}}\left(1+\frac{1}{p^{2}}+\frac{1}{p^{4}}+\cdots\right) \prod_{\substack{p \mid v \\
p \nmid u}}\left(1+\frac{1}{p^{2}}+\frac{1}{p^{4}}+\cdots\right) .
\end{aligned}
$$

Let

$$
S=1+\frac{2^{2}}{p^{2}}+\frac{3^{2}}{p^{4}}+\cdots .
$$

It is clear that

$$
S\left(1-\frac{1}{p^{2}}\right)^{2}=1+\frac{2}{p^{2}}\left(\frac{1}{1-\frac{1}{p^{2}}}\right) .
$$

Therefore,

$$
\begin{aligned}
\sum_{\substack{n=1 \\
(n, d)=1 \\
2 \nmid n}}^{\infty} \frac{r^{2}(n)}{n^{2}} & =\prod_{\substack{p \nmid u v \\
p \neq 2}}\left(1-\frac{1}{p^{2}}\right)^{-3}\left(1+\frac{1}{p^{2}}\right) \prod_{p \mid u v} \frac{p^{2}}{p^{2}-1} \prod_{p \mid d}\left(\frac{p^{2}}{p^{2}-1}\right)^{-1} \\
& =\frac{27 \zeta^{4}(2)}{80 \zeta(4)} \prod_{p \mid u v} \frac{\left(p^{2}-1\right)^{2}}{p^{2}\left(p^{2}+1\right)} \prod_{p \mid d}\left(\frac{p^{2}}{p^{2}-1}\right)^{-1} \\
& =\frac{3 \pi^{4}}{128} \prod_{p \mid u v} \frac{\left(p^{2}-1\right)^{2}}{p^{2}\left(p^{2}+1\right)} \prod_{p \mid d}\left(\frac{p^{2}}{p^{2}-1}\right)^{-1},
\end{aligned}
$$

where we used the identities $\zeta(2)=\pi^{2} / 6$ and $\zeta(4)=\pi^{4} / 90$. Now from (6)-(8), we get the assertion.

Lemma 7. Let $u$ and $v$ be odd integers with $(u, v)=d \geq 2$, and $\chi_{u}^{0}$ and $\chi_{v}^{0}$ be the principal characters modulo $u$ and $v$, respectively. Then for any integer $m \geq 0$ we have the asymptotic formula

$$
\begin{aligned}
& \sum_{\substack{\chi \bmod d \\
\chi(-1)=-1}} \chi\left(2^{m}\right)\left|L\left(1, \chi \chi_{u}^{0}\right)\right|^{2}\left|L\left(1, \chi \chi_{v}^{0}\right)\right|^{2} \\
& =\frac{(3 m+5) \pi^{4}}{72 \cdot 2^{m+1}} \phi(d) \frac{\prod_{p \mid u v} \frac{\left(p^{2}-1\right)^{2}}{p^{2}\left(p^{2}+1\right)}}{\prod_{p \mid d} \frac{p^{2}}{p^{2}-1}}+O_{m}\left(d^{\varepsilon}\right) .
\end{aligned}
$$


Proof. For convenience, we put

$$
A(y, \chi)=\sum_{N<n \leq y} \chi(n) r(n),
$$

where $N$ is a parameter with $d \leq N<d^{4}$ and $r(n)$ was defined in Lemma 6 . Then from Abel's identity we have

$$
\begin{aligned}
L\left(1, \chi \chi_{u}^{0}\right) L\left(1, \chi \chi_{v}^{0}\right) & =\sum_{n=1}^{\infty} \frac{\chi(n) r(n)}{n} \\
& =\sum_{1 \leq n \leq N} \frac{\chi(n) r(n)}{n}+\int_{N}^{\infty} \frac{A(y, \chi)}{y^{2}} d y .
\end{aligned}
$$

Hence, we can write

$$
\begin{aligned}
& \sum_{\substack{\chi \bmod d \\
\chi(-1)=-1}} \chi\left(2^{m}\right)\left|L\left(1, \chi \chi_{u}^{0}\right)\right|^{2}\left|L\left(1, \chi \chi_{v}^{0}\right)\right|^{2} \\
&=\sum_{\substack{\chi \bmod d \\
\chi(-1)=-1}} \chi\left(2^{m}\right)\left(\sum_{1 \leq n_{1} \leq N} \frac{\bar{\chi}\left(n_{1}\right) r\left(n_{1}\right)}{n_{1}}+\int_{N}^{\infty} \frac{A(y, \bar{\chi})}{y^{2}} d y\right) \\
&\left.\times \sum_{1 \leq n_{2} \leq N} \frac{\chi\left(n_{2}\right) r\left(n_{2}\right)}{n_{2}}+\int_{N}^{\infty} \frac{A(y, \chi)}{y^{2}} d y\right) \\
&= \sum_{\substack{\chi \bmod d \\
\chi(-1)=-1}} \chi\left(2^{m}\right)\left(\sum_{1 \leq n_{1} \leq N} \frac{\bar{\chi}\left(n_{1}\right) r\left(n_{1}\right)}{n_{1}}\right)\left(\sum_{1 \leq n_{2} \leq N} \frac{\chi\left(n_{2}\right) r\left(n_{2}\right)}{n_{2}}\right) \\
&+\sum_{\substack{\chi \bmod d \\
\chi(-1)=-1}} \chi\left(2^{m}\right)\left(\sum_{1 \leq n_{1} \leq N} \frac{\bar{\chi}\left(n_{1}\right) r\left(n_{1}\right)}{n_{1}}\right)\left(\int_{N}^{\infty} \frac{A(y, \chi)}{y^{2}} d y\right) \\
&+\sum_{\substack{\chi \bmod d \\
\chi(-1)=-1}} \chi\left(2^{m}\right)\left(\sum_{1 \leq n_{2} \leq N} \frac{\chi\left(n_{2}\right) r\left(n_{2}\right)}{n_{2}}\right)\left(\int_{N}^{\infty} \frac{A(y, \bar{\chi})}{y^{2}} d y\right) \\
&+\sum_{\substack{\chi \bmod d \\
\chi(-1)=-1}} \chi\left(2^{m}\right)\left(\int_{N}^{\infty} \frac{A(y, \bar{\chi})}{y^{2}} d y\right)\left(\int_{N}^{\infty} \frac{A(y, \chi)}{y^{2}} d y\right) \\
&:= M_{1}+M_{2}+M_{3}+M_{4} .
\end{aligned}
$$

Now we shall calculate each term in the expression (9). 
(i) From the orthogonality of Dirichlet characters we can write

$$
\text { (10) } \begin{aligned}
M_{1} & =\sum_{\substack{\chi \bmod d \\
\chi(-1)=-1}} \chi\left(2^{m}\right)\left(\sum_{1 \leq n_{1} \leq N} \frac{\bar{\chi}\left(n_{1}\right) r\left(n_{1}\right)}{n_{1}}\right)\left(\sum_{1 \leq n_{2} \leq N} \frac{\chi\left(n_{2}\right) r\left(n_{2}\right)}{n_{2}}\right) \\
= & \frac{\phi(d)}{2} \sum_{\substack{1 \leq n_{1} \leq N \\
2^{m} n_{2} \equiv n_{1}(\bmod d)}}^{\prime} \sum_{\substack{1 \leq n_{2} \leq N \\
n_{1}}}^{\prime} \frac{r\left(n_{1}\right) r\left(n_{2}\right)}{n_{1} n_{2}}-\frac{\phi(d)}{2} \sum_{\substack{1 \leq n_{1} \leq N \leq n_{2} \leq N \\
2^{m} n_{2} \equiv n_{1}(\bmod d)}}^{\prime} \frac{r\left(n_{1}\right) r\left(n_{2}\right)}{n_{1} n_{2}} .
\end{aligned}
$$

For convenience, we split the sum over $n_{1}$ or $n_{2}$ into the following cases: (a) $d \leq n_{1} \leq N, d / 2^{m} \leq n_{2} \leq N$; (b) $d \leq n_{1} \leq N, 1 \leq n_{2} \leq d / 2^{m}-1$; (c) $1 \leq n_{1} \leq d-1, d / 2^{m} \leq n_{2} \leq N$; (d) $1 \leq n_{1} \leq d-1,1 \leq n_{2} \leq d / 2^{m}-1$. So we have

$$
\begin{aligned}
& \frac{\phi(d)}{2} \sum_{\substack{d \leq n_{1} \leq N \\
2^{m} n_{2} \equiv n_{1}(\bmod d)}}^{\prime} \sum_{\substack{m \\
\text { m }}}^{\prime} \frac{r\left(n_{1}\right) r\left(n_{2}\right)}{n_{1} n_{2}} \\
& \ll \phi(d) \sum_{1 \leq s_{1} \leq N / d} \sum_{1 \leq s_{2} \leq 2^{m} N / d} \sum_{\substack{l_{1}=1 \\
l_{2} \equiv l_{1}(\bmod d)}}^{d-1} \sum_{l_{2}=1}^{d-1} \frac{r\left(s_{1} d+l_{1}\right) r\left(s_{2} d+l_{2}\right)}{\left(s_{1} d+l_{1}\right)\left(s_{2} d+l_{2}\right)} \\
& \ll \phi(d) \sum_{1 \leq s_{1} \leq N / d} \sum_{1 \leq s_{2} \leq 2^{m}} \sum_{N / d}^{d-1} \frac{\left[\left(s_{1} d+l_{1}\right)\left(s_{2} d+l_{1}\right)\right]^{\varepsilon}}{\left(s_{1} d+l_{1}\right)\left(s_{2} d+l_{1}\right)} \\
& \ll \frac{\phi(d)}{d} \sum_{1 \leq s_{1} \leq N / d} \sum_{1 \leq s_{2} \leq 2^{m} N / d} \frac{\left[\left(s_{1} d+1\right)\left(s_{2} d+1\right)\right]^{\varepsilon}}{s_{1} s_{2}} \\
& \ll_{m} d^{\varepsilon}
\end{aligned}
$$

and

$$
\frac{\phi(d)}{2} \sum_{\substack{d \leq n_{1} \leq N \\ 2^{m} n_{2} \equiv n_{1}(\bmod d)}}^{\prime} \sum_{\substack{1 \leq n_{2} \leq d / 2^{m}-1 \\ n_{1} n_{2}}}^{\prime} \frac{r\left(n_{1}\right) r\left(n_{2}\right)}{n^{2}}
$$

$$
\ll \phi(d) \sum_{1 \leq r \leq N / d} \sum_{1 \leq n_{2} \leq d / 2^{m}-1}\left(r n_{2} d\right)^{\varepsilon-1} \ll d^{\varepsilon},
$$

and also

$$
\frac{\phi(d)}{2} \sum_{\substack{1 \leq n_{1} \leq d-1 \\ 2^{m} n_{2} \equiv n_{1}(\bmod d)}}^{\prime} \sum_{2^{m} \leq n_{2} \leq N}^{\prime} \frac{r\left(n_{1}\right) r\left(n_{2}\right)}{n_{1} n_{2}} \ll d^{\varepsilon},
$$

where we have used the estimate $r(n) \ll n^{\varepsilon}$.

For the case $1 \leq n_{1} \leq d-1,1 \leq n_{2} \leq d / 2^{m}-1$, the solution of the 
congruence $2^{m} n_{2} \equiv n_{1}(\bmod d)$ is $2^{m} n_{2}=n_{1}$. Hence,

$$
\begin{aligned}
\frac{\phi(d)}{2} & \sum_{\substack{1 \leq n_{1} \leq d-1 \leq n_{2} \leq d / 2^{m}-1 \\
2^{m} n_{2} \equiv n_{1}(\bmod d)}}^{\prime} \frac{r\left(n_{1}\right) r\left(n_{2}\right)}{n_{1} n_{2}} \\
& =\frac{\phi(d)}{2^{m+1}} \sum_{1 \leq n_{2} \leq d / 2^{m}-1}^{\prime} \frac{r\left(2^{m} n_{2}\right) r\left(n_{2}\right)}{n_{2}^{2}}=\frac{\phi(d)}{2^{m+1}} \sum_{n=1}^{\infty} \frac{r\left(2^{m} n\right) r(n)}{n^{2}}+O_{m}\left(d^{\varepsilon}\right) .
\end{aligned}
$$

Now from Lemma 6, we immediately get

$$
\begin{gathered}
\frac{\phi(d)}{2} \sum_{\substack{1 \leq n_{1} \leq N \\
2^{m} n_{2} \equiv n_{1}(\bmod d)}}^{\prime} \sum_{1 \leq n_{2} \leq N}^{\prime} \frac{r\left(n_{1}\right) r\left(n_{2}\right)}{n_{1} n_{2}} \\
=\frac{(3 m+5) \pi^{4}}{72 \cdot 2^{m+1}} \phi(d) \frac{\prod_{p \mid u v} \frac{\left(p^{2}-1\right)^{2}}{p^{2}\left(p^{2}+1\right)}}{\prod_{p \mid d} \frac{p^{2}}{p^{2}-1}}+O_{m}\left(d^{\varepsilon}\right) .
\end{gathered}
$$

Similarly, we also get the estimate

$$
\begin{aligned}
& \frac{\phi(d)}{2} \sum_{\substack{1 \leq n_{1} \leq N \leq n_{2} \leq N \\
2^{m} n_{2} \equiv-n_{1}(\bmod d)}}^{\prime} \frac{r\left(n_{1}\right) r\left(n_{2}\right)}{n_{1} n_{2}} \\
& =\frac{\phi(d)}{2} \sum_{\substack{1 \leq n_{1} \leq N \\
2^{m} n_{2}+n_{1}=d}}^{\prime} \sum_{\substack{1 \leq n_{2} \leq N \\
n_{1}}}^{\prime} \frac{r\left(n_{1}\right) r\left(n_{2}\right)}{n_{1} n_{2}}+\frac{\phi(d)}{2} \sum_{\substack{1 \leq n_{1} \leq N \\
2^{m} n_{2}+n_{1}=l d, l \geq 2}}^{\prime} \sum_{\substack{1 \leq n_{2} \leq N \\
l}}^{\prime} \frac{r\left(n_{1}\right) r\left(n_{2}\right)}{n_{1} n_{2}} \\
& \ll \phi(d) \sum_{1 \leq n \leq d-1} \frac{2^{m} r(n) r\left(\frac{d-n}{2^{m}}\right)}{n(d-n)}+\phi(d) \sum_{1 \leq n_{1} \leq N}^{\prime} \sum_{l=\left[n_{1} / d\right]+2}^{\left[\left(N+n_{1}\right) / d\right]} \frac{2^{m} r\left(n_{1}\right) r\left(\frac{l d-n_{1}}{2^{m}}\right)}{l d n_{1}-n_{1}^{2}} \\
& \ll_{m} \frac{\phi(d)}{d} \sum_{1 \leq n \leq d-1} \frac{(n(d-n))^{\varepsilon}}{n}+\frac{\phi(d)}{d} \sum_{1 \leq n_{1} \leq N}^{\prime} \sum_{l=\left[n_{1} / d\right]+2}^{\left[\left(N+n_{1}\right) / d\right]} \frac{n_{1}^{\varepsilon}\left(l d-n_{1}\right)^{\varepsilon}}{l n_{1}-n_{1}^{2} / d} \\
& \ll_{m} d^{\varepsilon}+\frac{\phi(d) d^{\varepsilon}}{d} \sum_{n_{1}=1}^{N} \sum_{l=1}^{N} \frac{n_{1}^{\varepsilon} l^{\varepsilon}}{l n_{1}} \ll_{m} d^{\varepsilon} .
\end{aligned}
$$

Then from (10)-(12), we have

$$
M_{1}=\frac{(3 m+5) \pi^{4}}{72 \cdot 2^{m+1}} \phi(d) \frac{\prod_{p \mid u v} \frac{\left(p^{2}-1\right)^{2}}{p^{2}\left(p^{2}+1\right)}}{\prod_{p \mid d} \frac{p^{2}}{p^{2}-1}}+O_{m}\left(d^{\varepsilon}\right) .
$$


(ii) Note the partition identity

$$
\begin{aligned}
A(y, \chi)= & \sum_{n \leq \sqrt{y}} \chi(n) \chi_{u}^{0}(n) \sum_{m \leq y / n} \chi(m) \chi_{v}^{0}(m) \\
& +\sum_{m \leq \sqrt{y}} \chi(m) \chi_{v}^{0}(m) \sum_{n \leq y / m} \chi(n) \chi_{u}^{0}(n) \\
& -\sum_{n \leq \sqrt{N}} \chi(n) \chi_{u}^{0}(n) \sum_{m \leq N / n} \chi(m) \chi_{v}^{0}(m) \\
& -\sum_{m \leq \sqrt{N}} \chi(m) \chi_{v}^{0}(m) \sum_{n \leq N / m} \chi(n) \chi_{u}^{0}(n) \\
& -\left(\sum_{n \leq \sqrt{y}} \chi(n) \chi_{u}^{0}(n)\right)\left(\sum_{n \leq \sqrt{y}} \chi(n) \chi_{v}^{0}(n)\right) \\
& +\left(\sum_{n \leq \sqrt{N}} \chi(n) \chi_{u}^{0}(n)\right)\left(\sum_{n \leq \sqrt{N}} \chi(n) \chi_{v}^{0}(n)\right) .
\end{aligned}
$$

Applying the Cauchy inequality and the estimates for character sums

$$
\begin{aligned}
\sum_{\chi \neq \chi_{0}}\left|\sum_{N \leq n \leq M} \chi(n)\right|^{2} & =\sum_{\chi \neq \chi_{0}}\left|\sum_{N \leq n \leq M \leq N+d} \chi(n)\right|^{2} \\
=\phi(d) & \sum_{N \leq n \leq M \leq N+d} \chi_{0}(n)-\left|\sum_{N \leq n \leq M \leq N+d} \chi_{0}(n)\right|^{2} \leq \frac{\phi^{2}(d)}{4}
\end{aligned}
$$

and the identity

$$
\sum_{N \leq n \leq M} \chi(n) \chi_{u}^{0}(n)=\sum_{d \mid u} \mu(d) \chi(d) \sum_{N / d \leq n \leq M / d} \chi(n),
$$

we have

$$
\begin{aligned}
\sum_{\substack{\chi \bmod d \\
\chi(-1)=-1}}|A(y, \chi)|^{2} \ll & \sqrt{y} \sum_{n \leq \sqrt{y}} \sum_{\substack{\chi \bmod d \\
\chi(-1)=-1}}\left|\sum_{m \leq y / n} \chi(m) \chi_{u}^{0}(m)\right|^{2} \\
& +\sqrt{y} \sum_{m \leq \sqrt{y}} \sum_{\substack{\chi \bmod d \\
\chi(-1)=-1}}\left|\sum_{n \leq y / m} \chi(n) \chi_{v}^{0}(n)\right|^{2} \\
& +\sum_{\substack{\chi \bmod d \\
\chi(-1)=-1}}\left|\sum_{n \leq \sqrt{y}} \chi(n) \chi_{u}^{0}(n)\right|^{2} \times\left|\sum_{n \leq \sqrt{y}} \chi(n) \chi_{v}^{0}(n)\right|^{2} \\
\ll & y d^{2+\varepsilon} .
\end{aligned}
$$


Then from the Cauchy inequality and (14) we can write

$$
\begin{aligned}
M_{2} & =\sum_{\substack{\chi \bmod d \\
\chi(-1)=-1}} \chi\left(2^{m}\right)\left(\sum_{1 \leq n_{1} \leq N} \frac{\bar{\chi}\left(n_{1}\right) r\left(n_{1}\right)}{n_{1}}\right)\left(\int_{N}^{\infty} \frac{A(y, \chi)}{y^{2}} d y\right) \\
& \ll \sum_{1 \leq n_{1} \leq N} n_{1}^{\varepsilon-1} \int_{N}^{\infty} \frac{1}{y^{2}}\left(\sum_{\chi(-1)=-1}|A(y, \chi)|\right) d y \\
& \ll N^{\varepsilon} \int_{N}^{\infty} \frac{d^{3 / 2+\varepsilon} \sqrt{y}}{y^{2}} d y \ll \frac{d^{3 / 2+\varepsilon}}{N^{1 / 2-\varepsilon}} .
\end{aligned}
$$

(iii) Similar to (ii), we can also get

$$
M_{3} \ll \frac{d^{3 / 2+\varepsilon}}{N^{1 / 2-\varepsilon}} .
$$

(iv) By the same argument as in (ii), and noting the absolute convergence of the integrals, we can write

$$
\text { (17) } \begin{aligned}
M_{4} & =\sum_{\substack{\chi \bmod d \\
\chi(-1)=-1}} \chi\left(2^{m}\right)\left(\int_{N}^{\infty} \frac{A(y, \bar{\chi})}{y^{2}} d y\right)\left(\int_{N}^{\infty} \frac{A(y, \chi)}{y^{2}} d y\right) \\
& \leq \int_{N}^{\infty} \int_{N}^{\infty} \frac{1}{y^{2} z^{2}} \sum_{\substack{\chi \bmod d \\
\chi(-1)=-1}}|A(y, \bar{\chi})||A(z, \chi)| d y d z \\
& \ll \int_{N}^{\infty} \frac{1}{y^{2}} \int_{N}^{\infty} \frac{1}{z^{2}}\left(\sum_{\substack{\chi \bmod d \\
\chi(-1)=-1}}|A(y, \bar{\chi})|^{2}\right)^{1 / 2}\left(\sum_{\substack{\chi \bmod d \\
\chi(-1)=-1}}|A(z, \chi)|^{2}\right)^{1 / 2} d y d z \\
& \ll\left(\int_{N}^{\infty} \frac{1}{y^{2}}\left(\sum_{\substack{\chi \bmod d \\
\chi(-1)=-1}}|A(y, \chi)|^{2}\right)^{1 / 2} d y\right)^{2} \\
& \ll\left(\int_{N}^{\infty} \frac{d^{1+\varepsilon}}{y^{3 / 2}} d y\right)^{2} \ll \frac{d^{2+\varepsilon}}{N} .
\end{aligned}
$$

Now, taking $N=d^{3}$, combining (9)-(17) we obtain the asymptotic formula of Lemma 7.

Lemma 8 ([9, Lemma 5]). Let $p$ be a prime, and $\alpha$ and $\beta$ be nonnegative integers with $\beta \geq \alpha$. Then 


$$
\begin{aligned}
\sum_{d_{1} \mid p^{\beta}} \sum_{d_{2} \mid p^{\alpha}} \frac{d_{1}^{2} d_{2}^{2}}{\phi\left(d_{1}\right) \phi\left(d_{2}\right)} \phi(d) \frac{\prod_{p \mid u v} \frac{\left(p^{2}-1\right)^{2}}{p^{2}\left(p^{2}+1\right)}}{\prod_{p \mid d} \frac{p^{2}}{p^{2}-1}} \\
=p^{3 \alpha} \frac{\left(1+\frac{1}{p}\right)^{2}-\frac{1}{p^{3 \alpha+1}}}{1+\frac{1}{p}+\frac{1}{p^{2}}}+\frac{\left(p^{2}-1\right)^{2} p^{2 \alpha}\left(p^{\beta}-p^{\alpha}\right)}{(p-1)^{2}\left(p^{2}+1\right)},
\end{aligned}
$$

where $d=\left(d_{1}, d_{2}\right)$ denotes the greatest common divisor of $d_{1}$ and $d_{2}$.

Lemma 9. Let $q$ be any odd integer with $q \geq 3$, and $\chi$ be a Dirichlet character modulo $q$. Then for any integer $m \geq 0$, we have the asymptotic formulas

$$
\begin{aligned}
& \sum_{\substack{\chi \bmod q \\
\chi(-1)=-1}} \bar{\chi}\left(2^{m}\right)\left|\sum_{r=1}^{q} r \chi(r)\right|^{4} \\
& \quad=\frac{(3 m+5) q^{3} \phi^{4}(q)}{72 \cdot 2^{m+1}} \prod_{p^{\alpha} \| q} \frac{\frac{(p+1)^{3}}{p\left(p^{2}+1\right)}-\frac{1}{p^{3 \alpha-1}}}{1+\frac{1}{p}+\frac{1}{p^{2}}}+O_{m}\left(q^{6+\varepsilon}\right)
\end{aligned}
$$

and

$$
\sum_{\substack{\chi \bmod 4 q \\ \chi(-1)=-1}}\left|\sum_{r=1}^{4 q} r \chi(r)\right|^{4}=\frac{488}{9} q^{3} \phi^{4}(q) \prod_{p^{\alpha} \|_{q}} \frac{\frac{(p+1)^{3}}{p\left(p^{2}+1\right)}-\frac{1}{p^{3 \alpha-1}}}{1+\frac{1}{p}+\frac{1}{p^{2}}}+O_{m}\left(q^{6+\varepsilon}\right) .
$$

Proof. We only prove the first formula; the second one can be proved by the same method. From Lemmas 5 and 4 we have

$$
\begin{aligned}
& \sum_{\substack{\chi \bmod q \\
\chi(-1)=-1}} \bar{\chi}\left(2^{m}\right)\left|\sum_{r=1}^{q} r \chi(r)\right|^{4} \\
& =q^{4} \phi(q) \sum_{d \mid q} \sum_{l \mid q} \mu(d) \mu(l) \sum_{a=1}^{q} S\left(2^{m} a, q / d\right) S(a, q / l) \\
& =q^{4} \phi(q) \sum_{d \mid q} \sum_{l \mid q} \mu(d) \mu(l) \sum_{a=1}^{q}\left(\frac{d}{\pi^{2} q} \sum_{u \mid q / d} \frac{u^{2}}{\phi(u)} \sum_{\substack{\chi \bmod u \\
\chi(-1)=-1}} \chi\left(2^{m} a\right)|L(1, \chi)|^{2}\right) \\
& \times\left(\frac{l}{\pi^{2} q} \sum_{v \mid q / l} \frac{v^{2}}{\phi(v)} \sum_{\substack{\chi \bmod v \\
\chi(-1)=-1}} \chi(a)|L(1, \chi)|^{2}\right)
\end{aligned}
$$




$$
\begin{aligned}
= & \frac{q^{2} \phi(q)}{\pi^{4}} \sum_{d \mid q} \sum_{l \mid q} \mu(d) \mu(l) d l \sum_{u \mid q / d} \sum_{v \mid q / l} \frac{u^{2} v^{2}}{\phi(u) \phi(v)} \\
& \times \sum_{\substack{\chi_{1} \bmod u \\
\chi_{1}(-1)=-1}} \sum_{\substack{\chi_{2} \bmod v \\
\chi_{2}(-1)=-1}} \sum_{a=1}^{\prime} \chi_{1}\left(2^{m} a\right) \chi_{2}(a)\left|L\left(1, \chi_{1}\right)\right|^{2}\left|L\left(1, \chi_{2}\right)\right|^{2} .
\end{aligned}
$$

For each character $\chi_{1}$ modulo $u$, it is clear that there exists one and only one $q_{1} \mid u$ with a unique primitive character $\chi_{q_{1}}^{1}$ modulo $q_{1}$ such that $\chi_{1}=\chi_{q_{1}}^{1} \chi_{u}^{0}$. Similarly, we also have $\chi_{2}=\chi_{q_{2}}^{2} \chi_{v}^{0}$, where $q_{2} \mid v$, and $\chi_{q_{2}}^{2}$ is a primitive character modulo $q_{2}$. Noting that $u \mid q$ and $q \mid k$, from the orthogonality of Dirichlet characters we have

$$
\begin{aligned}
\sum_{a=1}^{q} \chi_{1}(a) \chi_{2}(a) & =\sum_{a=1}^{q}\left[\chi_{q_{1}}^{1}(a) \chi_{q}^{0}(a)\right]\left[\chi_{q_{2}}^{2}(a) \chi_{q}^{0}(a)\right] \\
& = \begin{cases}\phi(q) & \text { if } q_{1}=q_{2} \text { and } \chi_{q_{1}}^{1}=\overline{\chi_{q_{2}}^{2}} \\
0 & \text { otherwise. }\end{cases}
\end{aligned}
$$

Let $d_{1}=(u, v)$. If $q_{1}=q_{2}$ and $\chi_{q_{1}}^{1}=\overline{\chi_{q_{2}}^{2}}$, then $\chi_{q_{1}}^{1} \chi_{d_{1}}^{0}$ is also a character modulo $d_{1}$. So from (18), (19) and Lemma 7 we get

$$
\begin{aligned}
& \sum_{\substack{\chi \bmod q \\
\chi(-1)=-1}} \bar{\chi}\left(2^{m}\right)\left|\sum_{r=1}^{q} r \chi(r)\right|^{4} \\
& =\frac{q^{2} \phi^{2}(q)}{\pi^{4}} \sum_{d \mid q} \sum_{l \mid q} \mu(d) \mu(l) d l \sum_{u \mid q / d} \sum_{v \mid q / l} \frac{u^{2} v^{2}}{\phi(u) \phi(v)} \\
& \times \sum_{\substack{\chi \bmod (u, v) \\
\chi(-1)=-1}} \chi\left(2^{m}\right)\left|L\left(1, \chi \chi_{u}^{0}\right)\right|^{2}\left|L\left(1, \chi \chi_{v}^{0}\right)\right|^{2} \\
& =\frac{q^{2} \phi^{2}(q)}{\pi^{4}} \sum_{d \mid q} \sum_{l \mid q} \sum_{u \mid q / d} \sum_{v \mid q / l} \frac{\mu(d) \mu(l) d l u^{2} v^{2}}{\phi(u) \phi(v)} \\
& \times\left\{\frac{(3 m+5) \pi^{4}}{72 \cdot 2^{m+1}} \phi((u, v)) \frac{\prod_{p \mid u v} \frac{\left(p^{2}-1\right)^{2}}{p^{2}\left(p^{2}+1\right)}}{\prod_{p \mid(u, v)} \frac{p^{2}}{p^{2}-1}}+O_{m}\left((u, v)^{\varepsilon}\right)\right\} \\
& =\frac{(3 m+5) q^{2} \phi^{2}(q)}{72 \cdot 2^{m+1}} \sum_{d \mid q} \sum_{l \mid q} \sum_{u \mid q / d} \sum_{v \mid q / l} \frac{\mu(d) \mu(l) d l u^{2} v^{2}}{\phi(u) \phi(v)} \phi((u, v)) \frac{\prod_{p \mid u v} \frac{\left(p^{2}-1\right)^{2}}{p^{2}\left(p^{2}+1\right)}}{\prod_{p \mid(u, v)} \frac{p^{2}}{p^{2}-1}} \\
& +O_{m}\left(q^{6+\varepsilon}\right) \text {. }
\end{aligned}
$$


For any multiplicative functions $f(u)$ and $g(v)$,

$$
\begin{aligned}
& \sum_{d \mid q} \sum_{l \mid q} \mu(d) \mu(l) d l \sum_{u \mid q / d} \sum_{v \mid q / l} f(u) g(v) \\
& =\prod_{p^{\alpha} \| q}\left[\sum_{u \mid p^{\alpha}} \sum_{v \mid p^{\alpha}} f(u) g(v)-2 p \sum_{u \mid p^{\alpha-1}} \sum_{v \mid p^{\alpha}} f(u) g(v)+p^{2} \sum_{u \mid p^{\alpha-1}} \sum_{v \mid p^{\alpha-1}} f(u) g(v)\right] .
\end{aligned}
$$

Now from Lemma 8 and the identity

$$
\begin{aligned}
& p^{3 \alpha} \frac{\left(1+\frac{1}{p}\right)^{2}-\frac{1}{p^{3 \alpha+1}}}{1+\frac{1}{p}+\frac{1}{p^{2}}}+p^{2} \cdot p^{3 \alpha-3} \frac{\left(1+\frac{1}{p}\right)^{2}-\frac{1}{p^{3 \alpha-2}}}{1+\frac{1}{p}+\frac{1}{p^{2}}} \\
&-2 p\left[p^{3 \alpha-3} \frac{\left(1+\frac{1}{p}\right)^{2}-\frac{1}{p^{3 \alpha-2}}}{1+\frac{1}{p}+\frac{1}{p^{2}}}+\frac{\left(p^{2}-1\right)^{2} p^{2 \alpha-2}\left(p^{\alpha}-p^{\alpha-1}\right)}{(p-1)^{2}\left(p^{2}+1\right)}\right] \\
&=\frac{p^{3 \alpha+1}\left(1-\frac{1}{p}\right)^{2}}{1+\frac{1}{p}+\frac{1}{p^{2}}}\left(\frac{(p+1)^{3}}{\left(p^{2}+1\right) p^{2}}-\frac{1}{p^{3 \alpha}}\right)
\end{aligned}
$$

we get

$$
\begin{aligned}
& \sum_{d \mid q} \sum_{l \mid q} \sum_{u \mid q / d} \sum_{v \mid q / l} \frac{\mu(d) \mu(l) d l u^{2} v^{2}}{\phi(u) \phi(v)} \phi((u, v)) \frac{\prod_{p \mid u v} \frac{\left(p^{2}-1\right)^{2}}{p^{2}\left(p^{2}+1\right)}}{\prod_{p \mid(u, v)} \frac{p^{2}}{p^{2}-1}} \\
& =\prod_{p^{\alpha} \| q}\left\{\sum_{d \mid p^{\alpha}} \sum_{l \mid p^{\alpha}} \mu(d) d \mu(l) l \sum_{u\left|p^{\alpha} / d v\right| p^{\alpha} / l} \sum_{\prod^{\prime}} \frac{u v}{\phi(u) \phi(v)} \phi((u, v)) \frac{p_{p_{1}^{2} \mid u v} \frac{\left.p_{1}^{2}-1\right)^{2}}{p_{1}^{2}\left(p_{1}^{2}+1\right)}}{\prod_{p_{1} \mid(u, v)} \frac{p_{1}^{2}}{p_{1}^{2}-1}}\right\} \\
& =q \phi^{2}(q) \prod_{p^{\alpha} \| q} \frac{\frac{(p+1)^{3}}{p\left(p^{2}+1\right)}-\frac{1}{p^{3 \alpha-1}}}{1+\frac{1}{p}+\frac{1}{p^{2}}} .
\end{aligned}
$$

Now Lemma 9 follows immediately from (20) and (21).

Lemma 10. Let $q>2$ be an odd integer. Then

$$
\begin{aligned}
& \sum_{\substack{\chi \bmod q \\
\chi(-1)=1 \\
\chi \neq \chi_{0}}}\left|\sum_{b=1}^{4 q} b \chi \chi_{4}(b)\right|^{4} \\
& \quad=\sum_{\substack{\chi \bmod 4 q \\
\chi(-1)=-1}}\left|\sum_{b=1}^{4 q} b \chi(b)\right|^{4}-256 \sum_{\substack{\chi \bmod q \\
\chi(-1)=-1}}|1-\chi(2)|^{4}\left|\sum_{b=1}^{q} b \chi(b)\right|^{4} .
\end{aligned}
$$


Proof. Let $\chi_{4}^{0}$ denote the principal character modulo 4 . Then

$$
\begin{aligned}
& \sum_{\substack{\chi \bmod q \\
\chi(-1)=1 \\
\chi \neq \chi_{0}}}\left|\sum_{b=1}^{4 q} b \chi \chi_{4}(b)\right|^{4} \\
& =\sum_{\substack{\chi \bmod 4 q \\
\chi(-1)=-1}}\left|\sum_{b=1}^{4 q} b \chi(b)\right|^{4}-\sum_{\substack{\chi \bmod q \\
\chi(-1)=-1}}\left|\sum_{b=1}^{4 q} b \chi \chi_{4}^{0}(b)\right|^{4} .
\end{aligned}
$$

For the inner summation in the second term, we have

$$
\begin{aligned}
\sum_{b=1}^{4 q} b \chi \chi_{4}^{0}(b) & =\sum_{\substack{b=1 \\
(b, 2)=1}}^{4 q} b \chi(b) \\
& =2 \sum_{\substack{b=1 \\
(b, 2)=1}}^{q} b \chi(b)+2 \sum_{\substack{b=1 \\
2 \mid b}}^{q} b \chi(b)+4 q \sum_{\substack{b=1 \\
2 \mid b}}^{q} \chi(b)+2 q \sum_{\substack{b=1 \\
(b, 2)=1}}^{q} \chi(b) \\
& =2 \sum_{b=1}^{q} b \chi(b)+2 q \sum_{\substack{b=1 \\
2 \mid b}}^{q} \chi(b) \\
& =2 \sum_{b=1}^{q} b \chi(b)+2 \chi(2) \sum_{b=1}^{(q-1) / 2} \chi(b) .
\end{aligned}
$$

Now from Lemma 1, we have

$$
\sum_{b=1}^{4 q} b \chi \chi_{4}^{0}(b)=(4-4 \chi(2)) \sum_{b=1}^{q} b \chi(b) .
$$

Combining this with (22), we get the lemma.

3. Proof of the theorem. In this section we complete the proof of the theorem. From Lemmas 2, 3 and 10, we can write

$$
\begin{aligned}
\sum_{\chi \neq \chi_{0}}\left|\sum_{a<q / 4} \chi(a)\right|^{4}= & \frac{1}{8^{4} q^{4}} \sum_{\substack{\chi \bmod q \\
\chi(-1)=1 \\
\chi \neq \chi_{0}}}\left|\sum_{a=1}^{4 q} a \chi \chi_{4}(a)\right|^{4} \\
& +\frac{1}{16 q^{4}} \sum_{\substack{\chi \bmod q \\
\chi(-1)=-1}}|2+\bar{\chi}(2)-\bar{\chi}(4)|^{4}\left|\sum_{a=1}^{q} a \chi(a)\right|^{4}
\end{aligned}
$$




$$
\begin{aligned}
= & \frac{1}{8^{4} q^{4}} \sum_{\substack{\chi \bmod 4 q \\
\chi(-1)=-1}}\left|\sum_{a=1}^{4 q} a \chi(a)\right|^{4}-\frac{1}{16 q^{4}} \sum_{\substack{\chi \bmod q \\
\chi(-1)=-1}}|1-\chi(2)|^{4}\left|\sum_{a=1}^{q} a \chi(a)\right|^{4} \\
& +\frac{1}{16 q^{4}} \sum_{\substack{\chi \bmod q \\
\chi(-1)=-1}}|2+\bar{\chi}(2)-\bar{\chi}(4)|^{4}\left|\sum_{a=1}^{q} a \chi(a)\right|^{4} \\
= & \frac{1}{8^{4} q^{4}} \sum_{\substack{\chi \bmod 4 q \\
\chi(-1)=-1}}\left|\sum_{a=1}^{4 q} a \chi(a)\right|^{4} \\
& +\frac{1}{16 q^{4}} \sum_{\substack{\chi \bmod q \\
\chi(-1)=-1}}[40+12 \chi(2)+12 \bar{\chi}(2)-24 \chi(4) \\
& -24 \bar{\chi}(4)-4 \chi(8)-4 \bar{\chi}(8) 4 \chi(16)+4 \bar{\chi}(16)]\left|\sum_{a=1}^{q} a \chi(a)\right|^{4} .
\end{aligned}
$$

Noting that

$$
\sum_{\substack{\chi \bmod q \\ \chi(-1)=-1}} \bar{\chi}\left(2^{m}\right)\left|\sum_{r=1}^{q} r \chi(r)\right|^{4}=\sum_{\substack{\chi \bmod q \\ \chi(-1)=-1}} \chi\left(2^{m}\right)\left|\sum_{r=1}^{q} r \chi(r)\right|^{4},
$$

from Lemma 9 we get

$$
\sum_{\chi \neq \chi_{0}}\left|\sum_{a<q / 4} \chi(a)\right|^{4}=\frac{21 \phi^{4}(q)}{256 q} \prod_{p^{\alpha} \| q} \frac{\frac{(p+1)^{3}}{p\left(p^{2}+1\right)}-\frac{1}{p^{3 \alpha-1}}}{1+\frac{1}{p}+\frac{1}{p^{2}}}+O\left(q^{2+\varepsilon}\right) .
$$

This completes the proof of the theorem.

Acknowledgments. The authors express their gratitude to the referee for very helpful and detailed comments.

\section{References}

[1] D. A. Burgess, On a conjecture of Norton, Acta Arith. 27 (1975), 265-267.

[2] -, Mean value of character sums, Mathematika 33 (1986), 1-5.

[3] - Mean value of character sums II, ibid. 34 (1987), 1-7.

[4] T. Funakura, On Kronecker's limit formula for Dirichlet series with periodic coefficients, Acta Arith. 55 (1990), 59-73.

[5] G. Pólya, Über die Verteilung der quadratische Reste und Nichtreste, Göttingen Nachr. 1918, 21-29.

[6] I. M. Vinogradov, On the distribution of residues and non-residues of powers, J. Phys.-Math. Soc. Perm 1 (1918), 94-96. 
[7] Z. F. Xu and W. P. Zhang, On the $2 k$ th power mean of the character sums over short intervals, Acta Arith. 121 (2006), 149-160.

[8] W. P. Zhang, On the mean values of Dedekind sums, J. Théorie Nombres 8 (1996), 429-442.

[9] W. P. Zhang, Z. B. Xu and Y. Yi, A problem of D. H. Lehmer and its mean square value formula, J. Number Theory 103 (2003), 197-213.

Department of Mathematics

Northwest University

Xi'an, Shaanxi, P.R. China

E-mail: zhefengxu@hotmail.com

zfxu@eyou.com

wpzhang@nwu.edu.cn

Received on 20.12.2006

and in revised form on 15.11.2007 